\title{
Accogliere uomini, accogliere parole. Lampedusa: una storia esemplare di ieri e di oggi.
}

\section{Giovanni Ruffino}

\section{(2) OpenEdition \\ Journals}

Edizione digitale

URL: http://journals.openedition.org/esp/1402

DOI: 10.4000/esp.1402

ISSN: 2532-0319

Editore

Centre d'Information sur l'Éducation Bilingue et Plurilingue

\section{Edizione cartacea}

Data di pubblicazione: 1 dicembre 2017

Paginazione: 11-18

ISSN: 1127-266X

Notizia bibliografica digitale

Giovanni Ruffino, « Accogliere uomini, accogliere parole. Lampedusa: una storia esemplare di ieri e di oggi. », Éducation et sociétés plurilingues [Online], 43 | 2017, Messo online il 01 février 2019, consultato il 19 septembre 2020. URL : http://journals.openedition.org/esp/1402 ; DOI : https://doi.org/10.4000/ esp.1402 


\section{ACCOGLIERE UOMINI, ACGOGLIERE PAROLE. LAMPEDUSA: UNA STORIA ESEMPLARE DI IERI E DI OGGI.}

\section{Giovanni RUFFINo}

Pour ceux qui viennent d'Afrique, Lampedusa est la porte d'entrée à l'Europe. À $200 \mathrm{~km}$ de la Sicile et encore moins de la Tunisie, le monde la connaît aujourd'hui pour la générosité de ses habitants qui accueillent les multitudes de migrants arrivant d'Afrique dans des circonstances dramatiques. L'auteur croit que cette capacité à accueillir peut s'expliquer entre autres par l'histoire de ce petit lambeau de terre du canal de Sicile. Si on considère la Méditerranée avec des yeux de linguiste, on se rend vite compte que ces petites îles méritent un discours spécifique. On ne peut parler de Lampedusa sans parler de la Sicile, et on ne peut parler de la Sicile sans parler de la Méditerranée.

Mots-clés: Lampedusa, accueil, Europe, histoire, linguistique, Méditerranée.

For those coming from Africa, Lampedusa is the front door to Europe. Less than $200 \mathrm{ks}$ from Sicily and even closer to Tunisia, it is known today the world over for the generosity of its inhabitants who welcome the multitudes of migrants arriving from Africa in dire circumstances. The author believes that the capacity to take in can be explained among others by the history of that small bit of land protruding into the canal of Sicily. If one looks at the Mediterranean with a linguist's eye, one realizes that these small islands deserve a special mention. One cannot talk of Lampedusa without speaking of Sicily, and one cannot talk of Sicily without speaking of the Mediterranean.

Keyzeords: Lampedusa, welcome, Europe, history, linguistics, Mediterranean.

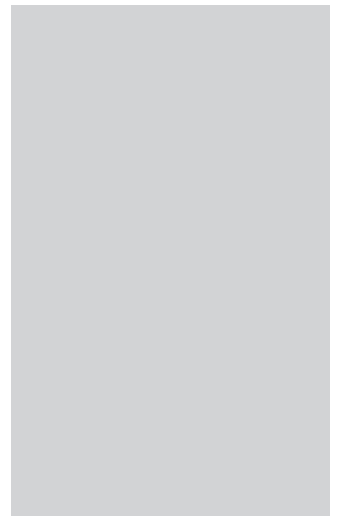

$\mathrm{P}$ er chi proviene dall'Africa, Lampedusa è la porta d'ingresso in Europa. Situata a $200 \mathrm{~km}$ dalla Sicilia e ancor meno dalla Tunisia, oggi è nota nel mondo per la generosità della sua gente nell'accogliere moltitudini di migranti provenienti dall'Africa in condizioni drammatiche. Io credo che questa disposizione all'accoglienza possa anche spiegarsi con la piccola storia di questo piccolo lembo di terra del Canale di Sicilia.

Se guardiamo al Mediterraneo con occhi da linguista, ci accorgiamo ben presto che le piccole isole meritano un discorso a parte: penso alle piccole isole della Sardegna - S. Antioco e S. Pietro -, alle isole della Campania, della Toscana, alle stesse Baleari e, infine, alle isole che circondano la Sicilia: le Eolie con Lipari, Ustica di fronte a Palermo, le Egadi con Favignana, e poi Pantelleria, Malta, le Pelagie con Lampedusa e Linosa. 
Accogliere uomini, accogliere parole.

Lampedusa: una storia esemplare di ieri e di oggi.

G. RufFino
Non si può parlare di Lampedusa senza parlare della Sicilia, e non si può parlare della Sicilia senza parlare del Mediterraneo.

Come ben si sa, la Sicilia è stata sin dall'antichità attraversata da correnti di lingua e cultura provenienti ora dal Nord (Normandia, Francia angioina), ora da ovest (Catalogna e Spagna), ora da Est (correnti bizantine e asiatiche), ora da Sud (Africa arabo-berbera). Lampedusa ne è partecipe di riflesso, ma la sua posizione linguistica ha una sua precisa fisionomia.

Se ci riferiamo alle correnti provenienti dall'Africa arabo-magrebina, Lampedusa e la Sicilia presentano condizioni analoghe. Ci sono casi in cui il dialetto lampedusano concorda con la sola Sicilia:

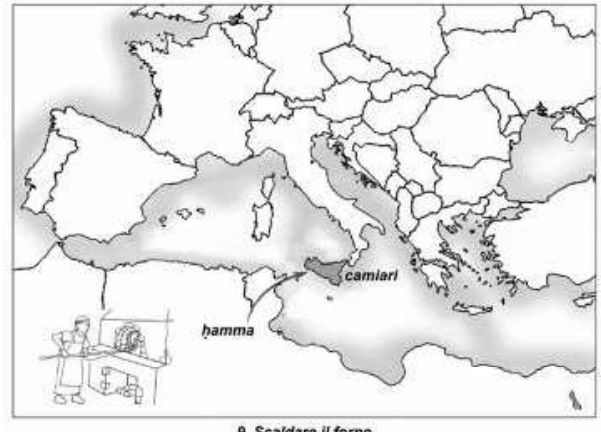

9. Scaldare il forno

con la Sicilia e l'Italia meridionale:

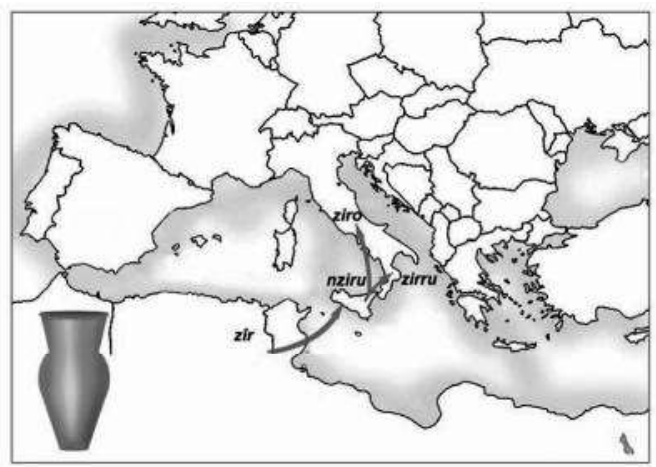




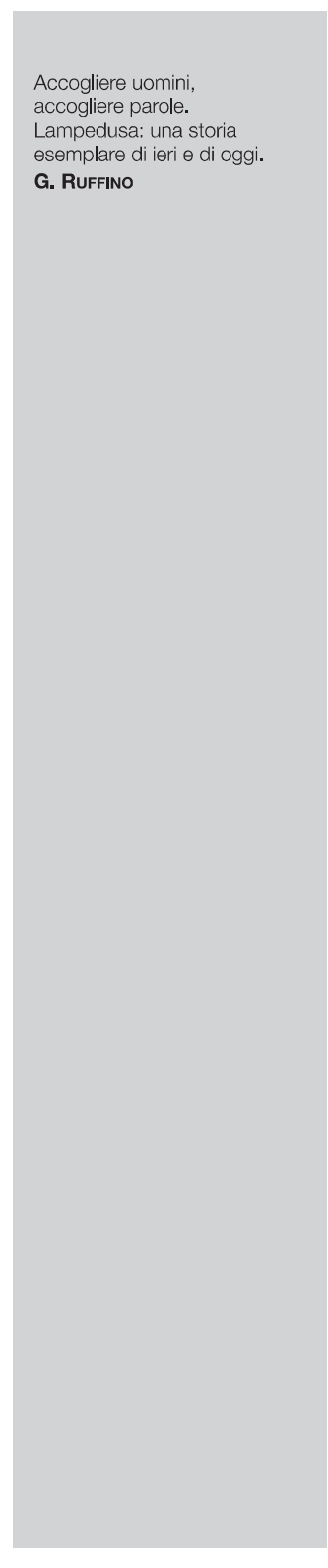

con l'intera penisola italiana:

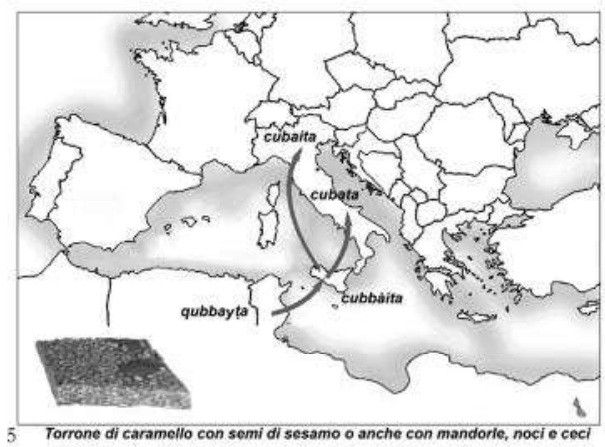

con la Sicilia e la Penisola iberica:

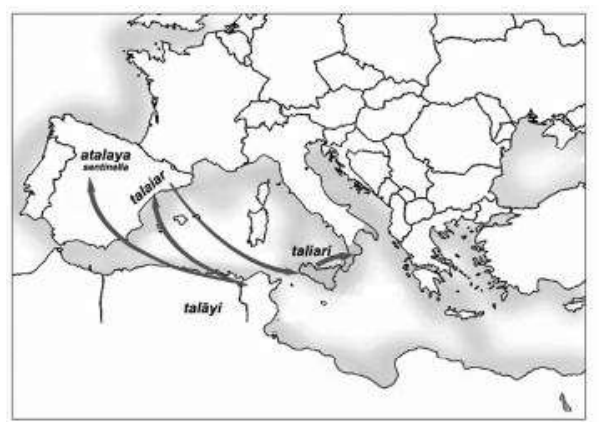

28. Guardare

con la Sicilia, l'Italia meridionale, la Sardegna e la Penisola iberica:

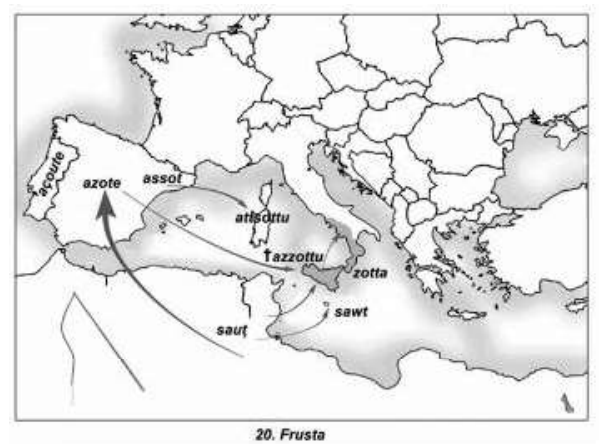


Accogliere uomini, accogliere parole. Lampedusa: una storia esemplare di ieri e di oggi.

G. RufFino
Ma al di là di queste corrispondenze areali, la situazione di Lampedusa ha una sua specificità, una singolare condizione determinata da ragioni storiche con conseguenze linguistiche.

Lampedusa è uno di quei luoghi in cui arrivi convinto di saperne abbastanza e da cui ti allontani carico di dubbi. Lampedusa è, in un certo modo, lo specchio in cui si riflettono contraddizioni e conflitti di questo nostro tempo, ma anche sintesi sorprendenti.

Nel passato, Lampedusa fu soprattutto un luogo di transito, priva di presenze abitative stabili, come rivelano i versi di Ludovico Ariosto (canto XL dell'Orlando Furioso), che fa di Lampedusa (Lipadusa) il teatro del decisivo duello tra Orlando, Oliviero e Brandimarte da un lato e Agramante, Sobrino e Gradasso dall'altro:

\section{D'abitazioni è l'isoletta vòta, piena d'umil mortelle e di ginepri ioconda solitudine e remota a cervi, a daini, a capriuoli, a lepri; e fuor ch'a piscatori, è poco nota, ove sovente a rimondati vepri sospendon per seccar l'umide reti: dormono intanto $i$ pesci in mar quieti.}

Terra di insediamenti più o meno effimeri (nordafricani, fenici, greci, romani, maltesi, siciliani e italiani del Sud). Terra di approdo e rifugio per cristiani in fuga dalle persecuzioni musulmane; per navi pirata, vascelli corsari, flotte crociate. Terra di eremiti di fedi diverse. Terra così remota da poterci impiantare una colonia penale dopo l'Unità d'Italia. Terra tanto vicina al continente africano da essere raggiunta da due missili libici al tempo di Gheddafi. Terra che alimenta ancora oggi due visioni contrapposte di una Lampedusa zona di frontiera da difendere e chiudere, e una Lampedusa, luogo in cui Europa e Africa s'incontrano, terra di confine da aprire a genti diverse, facendone laboratorio di cooperazione, di una visione mediterranea dell'Europa.

Ecco perché la prima cosa che colpisce chi arriva a Lampedusa, purché sappia ascoltare, è un groviglio di voci che si intrecciano e si sovrappongono.

In questo contesto sociale, i pescatori esercitano una speciale funzione etica per la solidità dei valori che incarnano. Valori che discendono dalla storia del popolamento dell'isola, fatta di convivenze sofferte eppure rapidamente assimilate, come dimostra il particolare dialetto lampedusano. 
Accogliere uomini, accogliere parole. Lampedusa: una storia esemplare di ieri e di oggi.

G. RufFino
E a questo proposito devo ricordare lo speciale saluto, tipico dei lampedusani: o cià! 'fiato mio!', che in Sicilia i genitori rivolgono ai loro bambini, ma che i lampedusani estendono all'intera cerchia amicale, e oltre.

Ma ora vado molto indietro con i miei personali ricordi e la mia esperienza di dialettologo.

Il mio primo viaggio a Lampedusa risale a oltre 40 anni fa, nell'imminenza di un congresso sulle condizioni linguistiche delle piccole isole del Mediterraneo ${ }^{1}$. Io $\square$ dialettologo alle prime armi $\square$ avevo programmato una relazione sul dialetto delle Pelagie, sicché dovetti esplorare la varietà lampedusana, allora del tutto sconosciuta. Sbarcando a Lampedusa avevo ben presente il quadro assai diversificato dei dialetti siciliani ${ }^{2}$, e andavo chiedendomi in quale tipologia linguistica avrei potuto includere quel dialetto.

Ascoltando e interrogando i lampedusani emergevano caratteristiche di un dialetto non classificabile all'interno del quadro delle varietà siciliane. Quello lampedusano era un dialetto nel quale convivevano caratteristiche trapanesi, palermitane, agrigentine, messinesi, eoliane, campane, persino nord-africane: un sincretismo linguistico non immaginabile, che aveva conquistato una sua stabilità?.

Tra le varietà dialettali conviventi a Lampedusa, spiccano le affinità con i dialetti messinesi, riconducibili a Lipari, nelle isole Eolie. Si intravede, inoltre, l'affiorare di certe isoglosse (che chiamerei micro-insulari) che congiungono Lipari a Lampedusa attraverso l'isola di Ustica ${ }^{4}$, le Egadi ${ }^{5}$ e in qualche caso Pantelleria ${ }^{6}$.

Ma a questo punto del nostro discorso occorre cercare di comprendere le speciali dinamiche che hanno determinato una situazione linguistica tanto singolare: occorre, cioè, risalire alle ragioni per le quali il dialetto di Lampedusa, nella sua struttura estremamente composita, si riconnetta a quelli che furono i momenti salienti del popolamento dell'arcipelago. Ed è anche singolare il fatto che un dialetto come quello lampedusano, che appare come il risultato dell'iniziale coesistenza di numerose varietà locali, si sia potuto stabilizzare in un tempo relativamente breve, come sistema comune di tutti i lampedusani. Ma bisogna pure dire che, ad accelerare tale processo di conguagliamento, avrà indubbiamente contribuito la grande coesione tra gli abitanti, in larga misura dediti ad una medesima attività lavorativa - la pesca -, tutti operanti entro gli stessi ristretti limiti spaziali, tutti solidali nella loro insularità.

Cos'era accaduto, dunque?

Era accaduto che il 18 settembre del 1843 due piroscafi della real 
Accogliere uomini, accogliere parole. Lampedusa: una storia esemplare di ieri e di oggi.

G. RufFino marina borbonica, il "Rondine" e "L’Antilope", salparono dal porto di Palermo alla volta di Lampedusa. Su quei piroscafi viaggiavano 120 persone - uomini, donne, bambini - destinate a colonizzare l'isola 7 . L'opera di colonizzazione ebbe subito inizio con la costruzione di alcune strade e di un caseggiato colonico. Negli anni successivi continuarono a giungere altri coloni allettati dal proclama reale che assicurava denaro, terra e casa. Fu anche impiantata una colonia di domiciliati coatti, provenienti da tutta la penisola italiana. Molti di costoro finirono con lo stabilirsi a Lampedusa dopo aver sposato ragazze del luogo: si devono ad essi anche alcuni tratti settentrionali assimilati dal dialetto, come palanca o fregna.

Ma il fatto più interessante e significativo è dato dai viaggi circumsiciliani che iniziarono nella metà del XVIII secolo: eoliani che, nel 1759, viaggiavano su quattro barconi verso Ustica, allora disabitata. E poi, negli anni successivi, verso l'isola di Marettimo, nelle Egadi, e infine a Lampedusa e a Pantelleria. Una circolazione determinata da pressione demografica, crisi economiche, epidemie, che esclude l'isola maggiore, la Sicilia - che dovette essere percepita come uno sterminato continente - e si concentra su percorsi microinsulari.

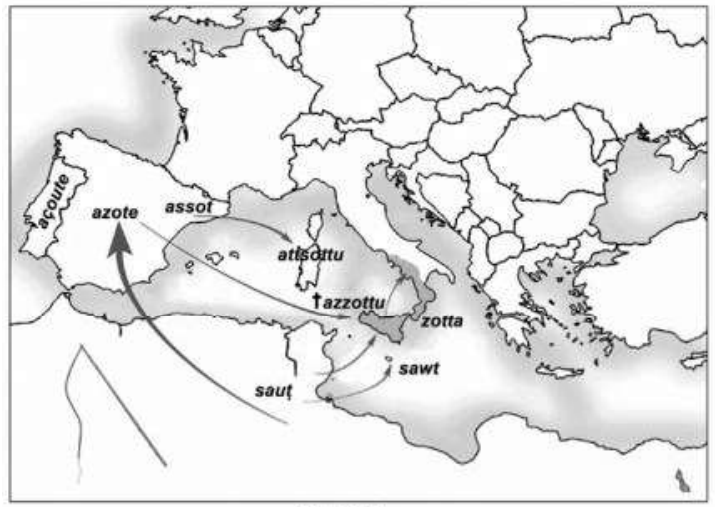

20. Frusta

Ritorno - per concludere - sulle riflessioni che proposi in occasione di uno degli ultimi Convegni della Società di Linguistica Italiana ${ }^{8}$ : «Sono convinto che la vicenda - ardua, sofferente - del popolamento 
Accogliere uomini, accogliere parole. Lampedusa: una storia esemplare di ieri e di oggi.

G. RufFino di Lampedusa possa contribuire a spiegare i tanti momenti di tolleranza e di apertura - l'umanità - dei lampedusani in un oggi ancor più arduo e lacerante, si manifesta anche nei giorni recenti. E malgrado tutto, si può dire che i discendenti dei "fuggitivi" di ieri guardano ai "fuggitivi" di oggi con occhio diverso da quello dei "continentali", impartendo lezioni memorabili anche alla "padania" opulenta.

Ancora una volta, le piccole isole si manifestano come luoghi aperti, nei quali il respiro ampio del mare prevale sulle angustie perimetrali, territoriali e anche mentali alimentate dal cinismo dei potenti, i quali pretenderebbero di farne ghetti orribili, prossimi - magari - alle ville sontuose il cui acquisto viene esibito alla stregua di miserabili slogan propagandistici. Angustie che non hanno però impedito nei secoli una sorta di migrazione microinsulare, che ha coinvolto Eolie e arcipelago campano, Ustica, Egadi, Pelagie, Pantelleria. E poi ancora le isole del medio e alto Tirreno. Migrazioni per mare, su affollati barconi, anche allora, che in qualche modo anticipano le drammatiche migrazioni intercontinentali di oggi.» 


\section{NOTE}

1. L'occasione era stata offerta dal VI Congresso internazionale dell'Atlante Linguistico Mediterraneo, tenuto a Palermo dal 7 all'11 ottobre 1975. In quella occasione presentai una relazione su Il dialetto di Lampedusa $e$ Linosa, con particolare riguardo alla terminologia mannaresca, poi pubblicato nel "Bollettino" del Centro di studi filologici e linguistici siciliani, 13, 1977, pp. 358-382.

2. Così come risulta dal saggio fondamentale di Giorgio Piccitto, La classificazione delle parlate siciliane e la metafonesi in Sicilia, in "Archivio storico della Sicilia orientale", IV, 1951, pp. 5-34. Il Piccitto aveva individuato le seguenti varietà areali: dialetti occidentali, distinti in dialetti trapanesi, palermitani e agrigentini occidentali; dialetti centrali, distinti in madoniti, nisseno-ennesi, agrigentini orientali; dialetti messinesi; dialetti catanesi-siracusani; dialetti sud-orientali.

3. Da una prima valutazione, su 34 tratti dialettali lampedusani esaminati, 6 richiamano le varietà siciliane occidentali, 7 quelle centrali, 11 quelle orientali, mentre 10 sono pansiciliani.

4. Cfr. - G. Ruffino, Migrazioni insulari e riflessi linguistici. Il caso di Ustica, in "Studi offerti a Girolamo Caracausi', Centro di studi filologici e linguistici siciliani, Palermo 1992, pp. 65-73.

5. Cfr. G. Ruffino, Le Egadi. Note storico-lingnistiche, in "Languages of the Mediterranean", a cura di G. Brincat, Malta 1994, pp. 150-154.

6. Fondamentali gli studi di Giovanni Tropea, condensati nel saggio lessicografico, pubblicato nel 1988 nella collana "Lessici siciliani" del Centro di studi filologici e linguistici siciliani, col titolo Lessico del dialetto di Pantelleria.

7. Una ricostruzione - con precisi riferimenti bibliografici - è contenuta in G. Ruffino, Il dialetto delle Pelagie e le inchieste dell'Atlante Linguistico Mediterraneo in Sicilia, Centro di studi filologici e linguistici siciliani, Palermo 1977, pp. 15-18.

8. G. Ruffino, Coesistenze e convergenze linguistiche nell'Italia pre- e post-unitaria, in "Coesistenze linguistiche nell'Italia pre- e postunitaria", Società di Linguistica Italiana, Bulzoni, Roma 2012, pp. 17-31, a p. 29. 